\title{
NOTE ON THE SEPARATION OF OLEIC ACID FROM OTHER FATTY ACIDS.
}

\author{
By J. Lewhowitsch, Ph.D.
}

(Read at the Meeting, February 7, 1900.)

In the course of a technical research on oleic acid, I had occasion to examine two methods which had been proposed recently by Twitchell and by Farnsteiner-the one for the separation of oleic acid (and other liquid acids) from solid fatty acids, the other for the separation of oleic acid from less saturated fatty acids.

I find the two methods practically useless for quantitative purposes, and, unpleasant though it be to publish results of this kind, I think some little service may be rendered to my colleagues by placing my results on record.

Twitchell's method is based on the insolubility in petroleum ether of the oleosulpho compound, obtained on treating a mixture of saturated and oleic acids with 85 per cent. sulphuric acid. He obtained apparently very good results in the case of a commercial oleic acid of a cold test of $12^{\circ} \mathrm{C}$., his method yielding a little over 7 per cent. solid acids.

I examined a commercial oleic acid made from tallow, having the solidifyingpoint of $8.5^{\circ} \mathrm{C}$. and the iodine value 82.27 , so that it may be accepted that the acid contained about 90 per cent. of true oleic acid, and practically very little of less saturated acids, if any.

I obtained in two experiments, when the petroleum ether was allowed to stand with the compound for a short time only, from $21 \cdot 6$ to 25 per cent. solid acids, and in two other experiments, where the mass was allowed to stand with the petroleum ether overnight, from $17 \cdot 8$ to $18 \cdot 7$ per cent. solid acids.

Another sample of oleic acid from the same source, having the iodine value 84.16 and hence containing about 7.25 per cent. solid fatty acids, gave by Twitchell's method from $35 \cdot 36$ to $44 \cdot 30$ per cent. solid acids. The best results were obtained by cooling the oleic acid in ice-water and allowing to stand with the acid for twelve hours before adding the petroleum ether. But even then, in four experiments, from 15.35 to 16.58 per cent. of soluble (supposed solid) acids were found, so that further attempts had to be abandoned.

Farnsteiner's method is based on the insolubility of barium oleate in cold benzene containing a little alcohol, in which menstruum the barium salts of the saturated acids are also insoluble. If this method yielded satisfactory results, an important step in the resolution of mixed fatty acids containing acids of the series $\mathrm{C}_{n} \mathrm{H}_{2 n} \mathrm{O}_{2}, \mathrm{C}_{n} \mathrm{H}_{2 \mathrm{n} \ldots 2} \mathrm{O}_{2}$, 
$\mathrm{C}_{11} \mathrm{H}_{2 \mathrm{n}-4} \mathrm{O}_{2}$, and less saturated would be made, as all the acids of greater unsaturation than oleic acid could be removed by means of crystallizing the barium salts from benzene containing the prescribed amount of alcohol. From the mixture of saturated acids and oleic acid then remaining, the oleic acid could be separated in a practically satisfactory manner by well-known methods.

I therefore examined samples of linseed, cottonseed, and olive oils. The result is set out in the table. The discordant results suggested the examination of oleic acid. The oleic acid of iodine value $84 \cdot 16$ was employed for the experiments. A glance at the table shows the hopelessness of the method.

TABLE I.

Results of Farnsteiner's Method.

\begin{tabular}{|c|c|c|c|c|c|c|c|c|c|}
\hline & & & & & Amol & it of Fatty & cids. & & \\
\hline & & & & & $\begin{array}{l}\text { 1st } \\
\text { Filtrate. }\end{array}$ & $\begin{array}{c}\text { 2nd } \\
\text { Filtrate. }\end{array}$ & $\begin{array}{l}\text { 3rd } \\
\text { Filtrate. }\end{array}$ & Insoluble. & found. \\
\hline Linseed oil & .. & $\ldots$ & $\cdots$ & $\ldots$ & $90 \cdot 30$ & $4 \cdot 71$ & 1.57 & $2 \cdot 54$ & $99 \cdot 12$ \\
\hline Linseed oil & $\ldots$ & $\ldots$ & $\ldots$ & $\ldots$ & $86 \cdot 38$ & $4 \cdot 55$ & 0.98 & $2 \cdot 43$ & $94 \cdot 34$ \\
\hline Linseed fatty & acids & $\ldots$ & $\ldots$ & $\ldots$ & $61 \cdot 62$ & $17 \cdot 20$ & $7 \cdot 80$ & 1.50 & $88 \cdot 12$ \\
\hline Linseed fatty & acids & $\ldots$ & $\ldots$ & $\cdots$ & $65 \cdot 32$ & $14 \cdot 02$ & $4 \cdot 40$ & $2 \cdot 80$ & $86 \cdot 54$ \\
\hline Olive oil & $\ldots$ & $\ldots$ & $\ldots$ & $\ldots$ & $28 \cdot 20$ & $26 \cdot 65$ & $10 \cdot 67$ & $24 \cdot 16$ & $89 \cdot 68$ \\
\hline Olive oil & $\ldots$ & $\ldots$ & $\ldots$ & $\ldots$ & $29 \cdot 17$ & $18 \cdot 65$ & $29 \cdot 30$ & $12 \cdot 12$ & $89 \cdot 77$ \\
\hline Olive oil & $\ldots$ & $\ldots$ & $\ldots$ & $\ldots$ & $33 \cdot 58$ & 22.55 & $18 \cdot 36$ & $11 \cdot 53$ & $86 \cdot 02$ \\
\hline Olive oil & & $\ldots$ & $\ldots$ & $\ldots$ & 31.93 & $21 \cdot 64$ & $24 \cdot 31$ & $12 \cdot 34$ & $90 \cdot 22$ \\
\hline Cottonseed oi & & $\ldots$ & $\ldots$ & $\ldots$ & $64 \cdot 36$ & $9 \cdot 58$ & $3 \cdot 43$ & $18 \cdot 86$ & $96 \cdot 23$ \\
\hline Cottonseed oi & & $\ldots$ & $\ldots$ & $\ldots$ & $61 \cdot 44$ & 10.57 & $3 \cdot 00$ & 19.95 & $94 \cdot 96$ \\
\hline Cottonseed oi & & $\ldots$ & $\ldots$ & $\ldots$ & $44 \cdot 25$ & $9 \cdot 81$ & $8 \cdot 80$ & 24.52 & $87 \cdot 38$ \\
\hline Cottonseed oi & & $\ldots$ & $\ldots$ & $\ldots$ & $44 \cdot 67$ & $12 \cdot 26$ & $7 \cdot 80$ & $22 \cdot 26$ & $86 \cdot 99$ \\
\hline Oleic acid & $\ldots$ & $\ldots$ & $\ldots$ & $\ldots$ & $85 \cdot 94$ & $8 \cdot 05$ & 3.00 & $2 \cdot 94$ & $99 \cdot 93$ \\
\hline Oleic acid & $\ldots$ & $\ldots$ & $\ldots$ & $\ldots$ & $85 \cdot 96$ & $5 \cdot 28$ & $2 \cdot 68$ & $3 \cdot 49$ & $97 \cdot 41$ \\
\hline Oleic a & $\ldots$ & $\ldots$ & $\ldots$ & $\ldots$ & $42 \cdot 84$ & $37 \cdot 74$ & $20 \cdot 18$ & - & $100 \cdot 76$ \\
\hline Oleic acid & $\ldots$ & $\ldots$ & $\ldots$ & $\ldots$ & $23 \cdot 28$ & $43 \cdot 74$ & $29 \cdot 43$ & $4 \cdot 20$ & $100 \cdot 65$ \\
\hline Oleic acid & $\ldots$ & $\ldots$ & $\ldots$ & $\ldots$ & $23 \cdot 70$ & $67 \cdot 37$ & $5 \cdot 96$ & $0 \cdot 44$ & $97 \cdot 47$ \\
\hline Oleic acid & $\ldots$ & $\ldots$ & $\ldots$ & $\ldots$ & $10 \cdot 68$ & $66 \cdot 26$ & $20 \cdot 12$ & $0 \cdot 40$ & $97 \cdot 46$ \\
\hline Oleic acid & $\ldots$ & $\ldots$ & $\ldots$ & $\ldots$ & $53 \cdot 73$ & $12 \cdot 51$ & $29 \cdot 24$ & $4 \cdot 70$ & $100 \cdot 58$ \\
\hline Oleic acid & $\ldots$ & $\ldots$ & $\ldots$ & $\cdots+$ & $47 \cdot 90$ & $15 \cdot 00$ & $34 \cdot 45$ & 0.63 & $99 \cdot 95$ \\
\hline
\end{tabular}

An explanation of my failure is found in the following table, which proves that, contrary to Farnsteiner's statement, barium oleate is not insoluble in benzene, and much less so in the proposed mixture of benzene and alcohol. 
TABLE II.

Barium Oleate, boiled $1 \frac{1}{2}$ hours under reflux condenser with 50 c.c. of Benzene, containing--

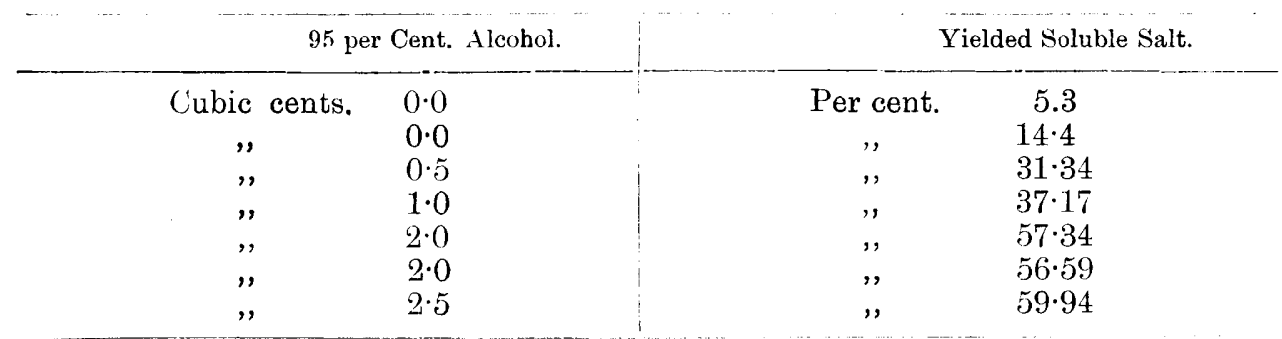

The experiments set out in the above table were carried out by Mr. C. D. Robertshaw, to whom my best thanks are due.

\section{Discussion.}

Mr. Hennen said that, on heating oleic acid with potassium hydroxide, one molecule of hydrogen was giveri off, whereas in the case of stearic acid and palmitic acid there was no evolution of hydrogen. It was to be presumed, also, that in the case of linolic acid, which contained one molecule of hydrogen less than oleic acid, no hydrogen would be given off on heating with potassium hydroxide. It would therefore seem that the hydrogen evolved on heating the mixture of fatty acids with potassium hydroxide ought to bear some relation to the quantity of oleic acid present, and might afford a means of determining it.

Mr. C. A. Mitchele inquired whether the author had made any experiments on the lines of another paper of Farnsteiner, in which lead elaidate was said to behave in petroleum spirit like the lead salts of the saturated fatty acids, though the results then obtained were not sufficiently constant for quantitative purposes. F. G. Edmed, in a recent paper before the Chemical Society, stated it to be possible, under certain circumstances, to convert oleic acid into elaïdic acid quantitatively, so that Farnsteiner's results in this direction, provided that they were reliable, might possibly now be of assistance in solving the problem.

Dr. Lewkowitsch said that Mr. Hehner's suggestion was certainly a valuable one, although the evolution of hydrogen could not be expected to be strictly quantitative so as to determine thereby pure oleic acid; and it would have to be shown that no evolution of hydrogen took place in the case of linolic acid. In reply to Mr. Mitchell, his own attempts to prepare elaidic acid on a considerable scale led him to think it hardly possible that Edmed could have really obtained, as he stated, 98 per cent. of pure elaidic acid. Also Farnsteiner had tried to convert quantitatively oleic acid into elaïdic, but had failed. 